\begin{tabular}{|c|l|}
\hline Title & Vibrational modes of GaA s hexagonal nanopillar arrays studied with ultrashort optical pul ses \\
\hline Author(s) & $\begin{array}{l}\text { Sakuma, Hirotaka; Tomoda, Motonobu; Otsuka, Paul H.; Matsuda, Osamu; W right, Oliver B.; Fukui, Takashi; } \\
\text { Tomioka, Katsuhiro; V eres, Istvan A. }\end{array}$ \\
\hline Citation & $\begin{array}{l}\text { A pplied Physics Letters, 100(13), 131902 } \\
\text { https://doi.org/10.1063/3696380 }\end{array}$ \\
\hline Issue Date & 2012-03-26 \\
\hline Doc URL & http://hdl.handle.net/2115/49118 \\
\hline Rights & $\begin{array}{l}\text { Copyright 2012 A merican Institute of Physics. This article may be downloaded for personal use only. A ny other use } \\
\text { requires prior permission of the author and the A merican Institute of Physics. The following article appeared in A ppl. } \\
\text { Phys. Lett. 100, 131902 (2012) and may be found at https://dx.doi.org/10.1063/3696380 }\end{array}$ \\
\hline Type & article \\
\hline File Information & APL100-13_131902.pdf \\
\hline
\end{tabular}

Instructions for use 


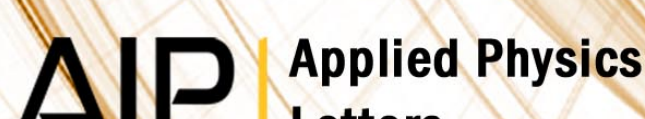 Letters}

\section{Vibrational modes of GaAs hexagonal nanopillar arrays studied with ultrashort optical pulses}

Hirotaka Sakuma, Motonobu Tomoda, Paul H. Otsuka, Osamu Matsuda, Oliver B. Wright et al.

Citation: Appl. Phys. Lett. 100, 131902 (2012); doi: 10.1063/1.3696380

View online: http://dx.doi.org/10.1063/1.3696380

View Table of Contents: http://apl.aip.org/resource/1/APPLAB/v100/i13

Published by the American Institute of Physics.

\section{Related Articles}

Surface acoustic wave band gaps in a diamond-based two-dimensional locally resonant phononic crystal for high frequency applications

J. Appl. Phys. 111, 014504 (2012)

Properties of defected one-dimensional terahertz plasmonic crystal films in a metal air-gap waveguide J. Appl. Phys. 110, 093101 (2011)

Vibrational and thermodynamic properties of $\beta-H M X$ : A first-principles investigation

J. Chem. Phys. 134, 204509 (2011)

Coupling of a surface plasmon with localized subwavelength microcavity modes

Appl. Phys. Lett. 98, 021105 (2011)

Evanescent modes in sonic crystals: Complex dispersion relation and supercell approximation

J. Appl. Phys. 108, 044907 (2010)

\section{Additional information on Appl. Phys. Lett.}

Journal Homepage: http://apl.aip.org/

Journal Information: http://apl.aip.org/about/about_the_journal

Top downloads: http://apl.aip.org/features/most_downloaded

Information for Authors: http://apl.aip.org/authors

\section{ADVERTISEMENT}




\title{
Vibrational modes of GaAs hexagonal nanopillar arrays studied with ultrashort optical pulses
}

\author{
Hirotaka Sakuma, ${ }^{1}$ Motonobu Tomoda, ${ }^{1}$ Paul H. Otsuka, ${ }^{1}$ Osamu Matsuda, ${ }^{1}$ \\ Oliver B. Wright, ${ }^{1, a)}$ Takashi Fukui, ${ }^{2}$ Katsuhiro Tomioka, ${ }^{2}$ and Istvan A. Veres ${ }^{3}$ \\ ${ }^{1}$ Division of Applied Physics, Graduate School of Engineering, Hokkaido University, Sapporo 060-8628, \\ Japan \\ ${ }^{2}$ Division of Electronics for Informatics, Graduate School of Information Science and Technology, Hokkaido \\ University, Sapporo 060-8628, Japan \\ ${ }^{3}$ Recendt, Research Center for Non-Destructive Testing GmbH, 4040 Linz, Austria
}

(Received 26 January 2012; accepted 1 March 2012; published online 26 March 2012)

\begin{abstract}
We investigate the vibrational modes of a triangular array of anisotropic, hexagonal GaAs nanopillars on a GaAs substrate through ultrafast changes in optical reflectivity. By comparison with simulations, we identify GHz resonances, mode shapes, and damping. In addition, by varying the pillar diameter, height, and pitch, we distinguish collective and localized modes. A proper understanding of substrate-attached nanostructure dynamics will lead to better characterization of nanosensors based on perturbations to vibrational resonances. (c) 2012 American Institute of Physics. [http://dx.doi.org/10.1063/1.3696380]
\end{abstract}

Understanding vibrations in nanoscale systems is important for the miniaturization of mechanical resonators and actuators, with applications in mass and gas sensing. ${ }^{1,2}$ Ultrafast optical techniques are well adapted for probing the GHz$\mathrm{THz}$ resonant frequencies involved. Most experiments have been carried out on metal nanostructures: A range of shapes have been investigated, such as spheres, ${ }^{3,4}$ cubes,,${ }^{5,6}$ discs or dots,${ }^{7,8}$ rods,${ }^{9,10}$ rings, ${ }^{11}$ or triangles. ${ }^{12}$ A smaller number of experiments has also been carried out on semiconductor nanostructures such as dots, ${ }^{13,14}$ pillars,${ }^{15}$ cantilevers, ${ }^{16}$ and spirals. ${ }^{17}$ All these experiments involve either single nanostructures or arrays. In spite of this intense interest, more research is required to better elucidate the effect of geometry on the resonances, especially for anisotropic nanostructures.

In this study, we investigate single-crystal GaAs nanopillars of hexagonal cross section arranged in a triangular array on a GaAs substrate using time-domain measurements of optical reflectivity. Unlike previous studies on nanopillars, our samples have lateral dimensions commensurate with their height, ensuring higher fundamental mode frequencies overall and efficient coupling to the substrate. We compare the Fourier spectrum of the reflectivity variation with numerical simulations based on a finite element technique, thereby elucidating the mode shapes and their damping as well as distinguishing between localized and collective modes.

Single crystal GaAs (111) nanopillars of hexagonal cross section, shown in Fig. 1, were prepared on a GaAs (111)B substrate in a triangular lattice array by selectivearea metallorganic vapor phase epitaxy (SA-MOVPE) ${ }^{18}$ After deposition of a $20 \mathrm{~nm}$ film of $\mathrm{SiO}_{2}$, electron beam lithography and wet chemical etching were used to make a periodic array of hexagonal openings. GaAs nanopillar growth was then carried out by epitaxy. The pillar height is $h=300 \pm 20 \mathrm{~nm}$, the pitch is $a=1000 \mathrm{~nm}$ and the pillar thickness is $d=330 \pm 10 \mathrm{~nm}$, as estimated by scanning elec-

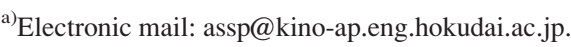

tron microscopy (SEM-see Fig. 1) over different regions of the sample.

As shown in Fig. 2(a), we use a mode-locked Ti:Sapphire laser of central wavelength $830 \mathrm{~nm}$, pulse duration $\sim 200 \mathrm{fs}$, and repetition rate $80 \mathrm{MHz}$ (period $12.5 \mathrm{~ns}$ ). The second harmonic at $415 \mathrm{~nm}$ is used for the pump beam (optical absorption depth $17 \mathrm{~nm}),{ }^{19}$ which is modulated at a frequency of $1 \mathrm{MHz}$ to allow lock-in detection. This beam of incident pulse energy $0.5 \mathrm{~nJ}$ is focused with linear polarization in the $x$ direction [see Fig. 1] at normal incidence to a full width at half maximum (FWHM) $\sim 5 \mu$ m diameter spot using a $\times 10$ objective lens. This leads to both steady state and transient temperature rises of $\sim 5 \mathrm{~K}$ and $\sim 80 \mathrm{~K}$, respectively. Nanopillar vibrations are induced by the thermoelastic and deformation potential mechanisms. ${ }^{20}$ The probe beam of wavelength $\lambda=830 \mathrm{~nm}$ (optical absorption depth $880 \mathrm{~nm})^{19}$ passes through a variable delay line and is focused with circular polarization at normal incidence to a similar spot size as the pump in order to monitor transient reflectivity changes $\sim 10^{-5}$ arising from displacements $\sim 10 \mathrm{pm}$ and strains $\sim 10^{-5}$. With our spot sizes, we are on average effectively exciting and probing $\sim 30$ nanopillars. All experiments are performed at room temperature.

Results for the relative reflectivity variation $\delta R(t) / R$ are shown in Fig. 2(b). Ultrafast carrier excitation produces a sharp positive spike at the moment of pump pulse arrival

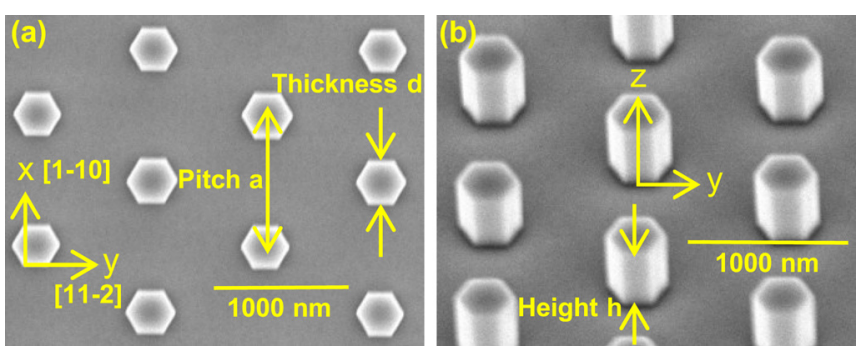

FIG. 1. Typical SEM images of the GaAs nanopillars. (a) Top view. (b) Oblique view. 

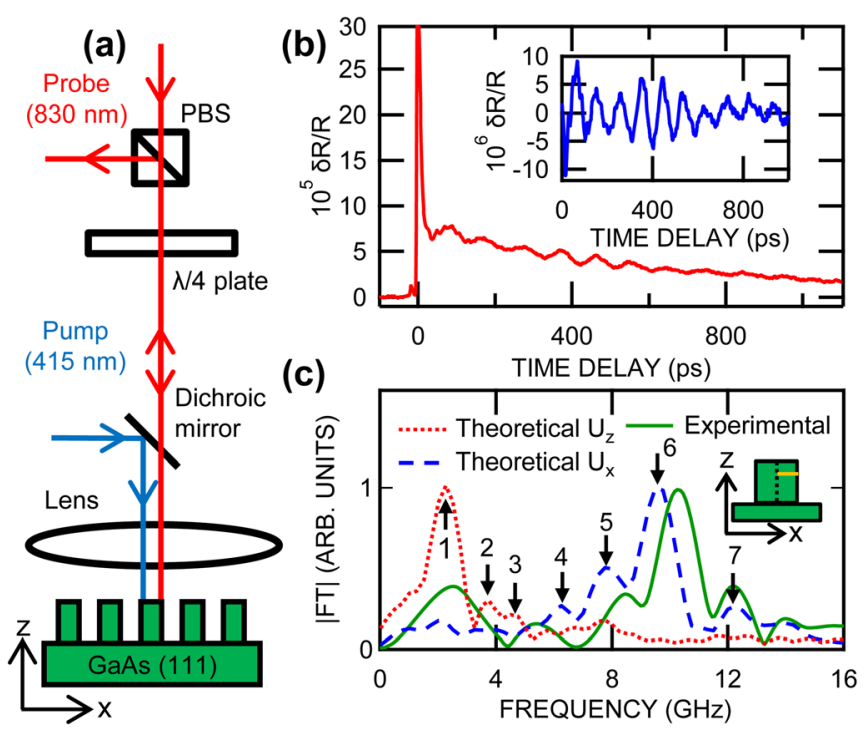

FIG. 2. (a) Experimental setup. PBS: Polarizing beam splitter. (b) Measured relative reflectivity variation $\delta R(t) / R$. Inset: Background subtracted data. (c) Moduli of the temporal Fourier transforms for $\delta R(t) / R$ (solid line) and for the simulated displacements: $U_{z}$ (dotted line) and $U_{x}$ (dashed line), both normalized (the latter enhanced by a factor of 9 ). The bright horizontal line segment in the inset was used to average the Fourier transform moduli.

$(t=0) . \delta R(t) / R$ then shows oscillations superimposed on a background variation arising from thermal diffusion, and are better viewed with the exponentially-fitted background subtracted [see inset of Fig. 2(b)]. They arise in general from both the shape changes of the vibrating nanopillars and the oscillating strain through photoelasticity.

We plot in Fig. 2(c) the modulus of the temporal Fourier transform of the background-subtracted data (solid curve). Vibrational resonance peaks are visible at 2.5, 5.4, 8.5, 10.2, 12.2, and $13.9 \mathrm{GHz}$. For quantitative data extraction, we apply least-squares fitting to the four highest peaks and to a Brillouin peak at $48.6 \mathrm{GHz}$ (Ref. 21) using a sum of five damped sinusoidal waves. The results for relative amplitude, Q-factor ( $\sim 10$ for the vibrational resonances), and phase lead $\psi$ are shown in Table I. (The parameters for the remaining smaller peaks could not be extracted accurately.) This fitting procedure is subject to errors of $\sim 10 \%$ (with the phase compared to $2 \pi$ ). Phase $\psi=0$ is commonly observed in the vibration of ultrashort-pulsed optical excitation of nanoobjects, ${ }^{3,15,22}$ characteristic of a displacive excitation mechanism. However, here there is significant thermal diffusion to the substrate, and so different values of the phase are recorded.

To visualize the vibrational modes, we implement a time-domain finite-element simulation (PZFlex, Weidlinger Associates, Inc.). A triangular array of nanopillars on a (111) GaAs substrate region of dimensions (in the $x, y$, and $z$ directions-see Fig. 1) $7.6 \times 7.0 \times 3.6 \mu \mathrm{m}^{3}$ was modeled. ${ }^{23,25} \mathrm{~A}$ downward vertical force field in the form of a half sine wave of duration 8.7 ps was applied uniformly over the top surface of all the pillars to approximately reproduce the experimental frequency spectrum.

Figure 2(c) also shows the simulated spectra $U_{x}$ and $U_{z}$ for the normalized $x$ - and $z$-directed displacements averaged over a line segment $3 / 4$ of the way up the pillar (see inset);
TABLE I. Fitting parameters for the experimental $\delta R(t) / R$ using a sum over five terms in the form $A_{i} \cos \left(2 \pi f_{i} t+\psi_{i}\right) \exp \left(-2 \pi f_{i} t / Q_{i}\right)$. (i)-(iv) refer to vibrations, whereas "Bulk" refers to the Brillouin peak whose Q-factor is determined by the optical penetration of probe beam.

\begin{tabular}{lllccc}
\hline \hline Mode & (i) & (ii) & (iii) & (iv) & Bulk \\
\hline Frequency $f_{i}(\mathrm{GHz})$ & 2.5 & 8.5 & 10.2 & 12.2 & 48.6 \\
Relative amplitude $A_{i}$ & 0.13 & 0.29 & 1.0 & 0.27 & 0.39 \\
Q-factor $Q_{i}$ & 7.5 & 8.9 & 15.8 & 30 & 16.4 \\
Phase $\left(\psi_{i} / \pi\right)$ & 0.28 & -0.32 & -0.66 & 0.96 & -0.67 \\
\hline \hline
\end{tabular}

resonant peaks at 2.2, 3.7, 4.5, 6.3, 7.5, 9.7, and $12.3 \mathrm{GHz}$ (modes 1-7) are indicated by arrows. The larger peaks 1, 5, 6 , and 7 correspond reasonably closely with the experimental modes (i)-(iv). Discrepancies in the peak positions presumably arise from local variations in pillar geometry, and smaller peaks may be lost in experiment owing to the associated inhomogeneous broadening. The experimental peak widths $(\propto 1 / Q)$, probably governed here mainly by radiation of acoustic waves to the bulk, agree overall with those of the simulation.

Figure 3(a) shows maps of the modulus of the temporal Fourier transforms $U_{x}$ and $U_{z}$ for 7 simulated resonances. The sections represent vertical cuts in the [ $1-10]$ direction [see dashed line in Fig. 3(a)] - hence the left-right symmetry. Deformations are shown in Fig. 3(b) and in animation elsewhere. $^{24}$ The different responses of $U_{x}$ and $U_{z}$ in Fig. 2(c) arise from the different mode shapes: For example, mode 1 at $2.2 \mathrm{GHz}$, corresponding to the vertical motion of the whole nanopillar, has a stronger $U_{z}$, whereas mode 6 at $9.7 \mathrm{GHz}$, corresponding to a coupled breathing-longitudinal mode, has a stronger $U_{x}$. For modes 1-4, the acoustic wavelength $\Lambda \sim 1 \mu \mathrm{m} \gg d$, leading, for our normal-incidence optical excitation, to a nearly uniform $U_{z}$ over the pillar top (see top views). Modes 5-7, in contrast, where $\Lambda$ is smaller, begin to show threefold symmetry from the anisotropy of GaAs (111).

To further elucidate these modes, we separately varied the pillar pitch $a$, width $d$, and height $h$ in the simulations as shown in Fig. 4. The frequencies of modes 1-3 decrease with increasing $a$ (mode 1 being approximately $\propto 1 / a$ ), whereas they are independent of $d$. In contrast, modes 5-7, which exhibit smaller displacements between the pillars [see Fig. 3(a)], show negligible dependence on $a$, but their frequencies are $\propto 1 / d$ [see dashed lines in Fig. 4(b)]. We conclude that modes 1-3 are collective modes of the array with wave vector $\mathbf{k}=0$ in the reduced-zone scheme, ${ }^{6,7}$ whereas $4-7$ are coupled breathing-longitudinal modes of localized character. Interestingly, mode 4 shows no dependence on $a$ and $d$ over the ranges considered. The spatial distribution of the displacements $U_{x}$ and $U_{z}$ for this mode are distinct from the other modes, as can be seen in Fig. 3(a). Mode frequency dependence on height $h$ only becomes apparent for modes 6 and 7, presumably because for them $\Lambda \sim 0.5 \mu \mathrm{m} \sim h$. The absence of an $h$ dependence of the frequency of mode 1 suggests that a simple mass and spring model, that would predict a $1 / \sqrt{h}$ variation, does not apply to this mode. The scaling of modes 2-3 compared to mode 1 is approximately that expected from an empty-lattice approximation ${ }^{6}$ : The 

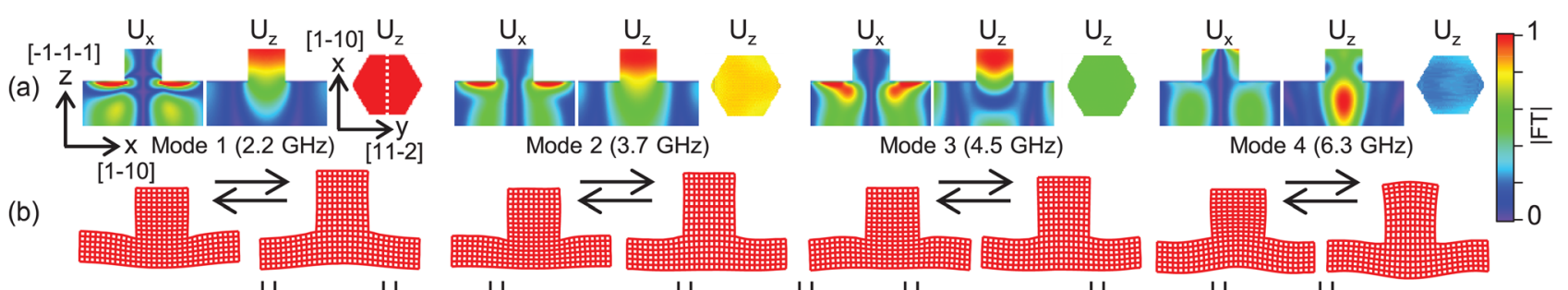

(a)
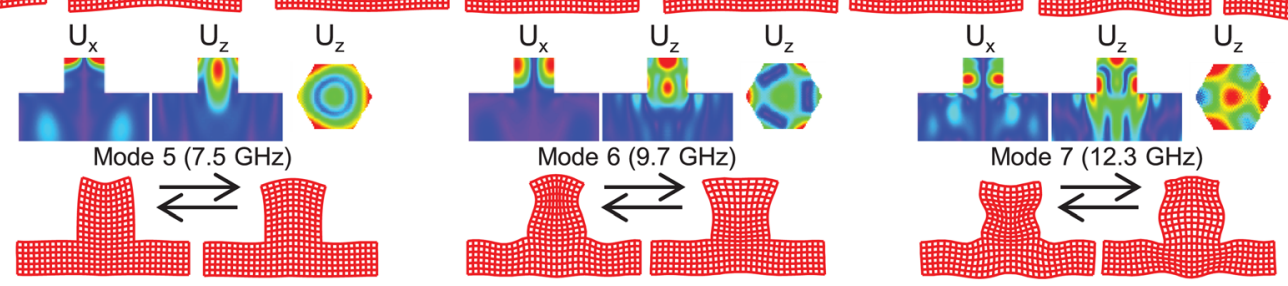

FIG. 3. (a) Simulated maps of the $x$ - and $z$-directed motion from moduli of the temporal Fourier transforms. The sections are vertical cuts in the [1 -10$]$ direction. (b) Sections of the mode deformations in anti-phase.

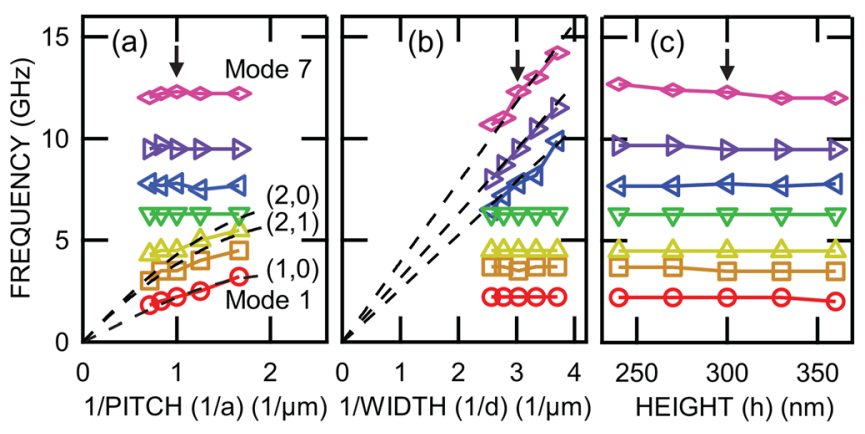

FIG. 4. The 7 simulated peak frequencies (a) vs. $1 / a$, with $d$ and $h$ fixed at the sample nominal values; (b) vs. $1 / d$ for constant $a$ and $h$; (c) vs. $h$ for constant $a$ and $w$. Arrows show nominal sample values. The dashed curves in (a) are fits, labelled by reciprocal lattice vector indices $(i, j)$ (Ref. 6), based on an empty-lattice model. The dashed curves in (b) are guides to the eye.

upper two dashed lines in Fig. 4(a) are predictions based on scaling by factors ${ }^{6}$ of $\sqrt{3}$ and 2 compared to data for mode 1 (fitted to a function in the form $c_{1} / a-c_{2} / a^{2}$, as shown by the lower dashed line).

This study elucidates the pillar mode-shapes and, through variations in pitch $a$, also identifies collective modes of the phononic structure at $\mathbf{k}=0$. In future, single-pillar excitation and localized detection would allow probing of the phononic dispersion relation at $\mathbf{k} \neq 0$. Combining our approach with lateral near-field scanning would open the way to mapping vibrational modes in anisotropic nanoscale structures and sensors in two-dimensions.

${ }^{1}$ Y. T. Yang, C. Callegari, X. L. Feng, K. L. Ekinci, and M. L. Roukes, Nano Lett. 6, 583 (2006).

${ }^{2}$ M. Li, H. X. Tang, and M. L. Roukes, Nat. Nanotechnol. 2, 114 (2007).

${ }^{3}$ N. Del Fatti, C. Voisin, D. Christofilos, F. Vallée, and C. Flytzanis, J. Chem. Phys. A 104, 4321 (2000).

${ }^{4}$ W. Huang, W. Qian, P. K. Jain, and M. A. El-Sayed, Nano Lett. 7, 3227 (2007).

${ }^{5}$ H. Petrova, C. H. Lin, S. de Liejer, M. Hu, J. M. McLellan, A. R. Siekkinen, B. J. Wiley, M. Marquez, Y. Xia, J. E. Sader et al., J. Chem. Phys. 126, 094709 (2007).
${ }^{6}$ J. F. Robillard, A. Devos, I. Roch-Jeune, and P. A. Mante, Phys. Rev. B 78, 064302 (2008).

${ }^{7}$ C. Giannetti, B. Revaz, F. Banfi, M. Montagnese, G. Ferrini, F. Cilento, S. Maccalli, P. Vavassori, G. Oliviero, E. Bontempi et al., Phys. Rev. B 76, 125413 (2007).

${ }^{8}$ H.-N. Lin, H. J. Maris, L. B. Freund, K. Y. Lee, H. Luhn, and D. P. Kern, J. Appl. Phys. 73, 37 (1993).

${ }^{9}$ M. Hu, X. Wang, G. V. Hartland, P. Mulvaney, J. P. Juste, and J. E. Sader, J. Am. Chem. Soc. 125, 14925 (2003).

${ }^{10}$ P. Zijlstra, A. L. Tchebotareva, J. W. M. Chon, M. Gu, and M. Orrit, Nano Lett. 8, 3493 (2008).

${ }^{11}$ T. A. Kelf, Y. Tanaka, O. Matsuda, E. M. Larsson, D. S. Sutherland, and O. B. Wright, Nano Lett. 11, 3893 (2011).

${ }^{12}$ J. Burgin, P. Langot, N. Del Fatti, F. Vallée, W. Huang, and M. A. ElSayed, J. Phys. Chem. C 112, 11231 (2008).

${ }^{13}$ T. D. Krauss and F. W. Wise, Phys. Rev. B 55, 9860 (1997).

${ }^{14}$ D. M. Mittleman, R. W. Schoenlein, J. J. Shiang, V. L. Colvin, A. P. Alivisatos, and C. V. Shank, Phys. Rev. B 49, 14435 (1994).

${ }^{15}$ S. O. Mariager, D. Khakhulin, H. T. Lemke, K. S. Kjær, L. Guerin, L. Nuccio, C. B. Sørensen, M. M. Nielsen, and R. Feidenhans, Nano Lett. 10, 2461 (2010)

${ }^{16}$ N. Liu, F. Giesen, M. Belov, J. Losby, J. Moroz, A. E. Fraser, G. McKinnon, T. J. Clement, V. Sauer, W. K. Hiebert et al., Nat. Nanotechnol. 3, 715 (2008).

${ }^{17}$ M. Yamaguchi, J. Liu, D. Ye, and T. M. Lu, J. Appl. Phys. 106, 033517 (2009).

${ }^{18}$ J. Noborisaka, J. Motohisa, and T. Fukui, Appl. Phys. Lett. 86, 213102 (2005).

${ }^{19}$ E. D. Palik and G. Ghosh, Electronic Handbook of Optical Constants of Solids (SciVision, Academic, New York, 1999).

${ }^{20}$ O. B. Wright, B. Perrin, O. Matsuda, and V. E. Gusev, Phys. Rev. B 64, 081202 (2001).

${ }^{21}$ Brillouin oscillations at frequency $f_{b}$ arise from the probe light reflected from longitudinal strain pulses in the substrate (Ref. 8): $f_{b}=2 n v_{l} / \lambda$, where $n=3.7$ is refractive index of GaAs at $\lambda=830 \mathrm{~nm}$ (Ref. 19) and $v_{l}=5.44$ $\mathrm{km} / \mathrm{s}$ is the [111] longitudinal sound velocity in GaAs (Ref. 25). The calculated $f_{b}=48.4 \mathrm{GHz}$ agrees with experiment. Changing $\lambda$ changed $f_{b}$ as expected.

${ }^{22}$ J. H. Hodak, A. Henglein, and G. V. Hartland, J. Chem. Phys 111, 8613 (1999).

${ }^{23}$ The $20 \mathrm{~nm}$ silica film was not included. Absorbing boundary conditions were used except for the free top surface. The element size was $5.7 \mathrm{~nm}^{3}$, the time step was $0.58 \mathrm{ps}$, and the simulation time was $1 \mathrm{~ns}$. Density $\rho=5.31 \mathrm{~g} / \mathrm{cm}^{3}$ and elastic constants $C_{11}=118.8, C_{12}=53.7$, and $C_{44}=59.4 \mathrm{GPa}$ of bulk GaAs were used (Ref. 25).

${ }^{24}$ See supplementary material at http://dx.doi.org/10.1063/1.3696380 for animations.

${ }^{25}$ D. Lide, CRC Handbook of Chemistry and Physics (CRC, Boca Raton, 1999). 